\title{
Enhancement of Autonomic Stress Response and Reduction of Subjective Stress by Lavender Inhalation During a Short-term Calculation Task
}

\author{
Shusaku Nomura, , , Kento MaEyama, ${ }^{*}$ Kanetoshi Iто **
}

\begin{abstract}
We investigated the effects of two prominent aromas, lavender and jasmine, on peripheral and cardiac autonomic nervous system activity under stressful conditions, in a highly reproducible manner using an olfactometer. The subjects comprised 17 healthy men aged 20-24 years. In this within-subjects study design, all subjects were required to perform a simple calculation task for 30 min to induce cognitive stress, and aroma from lavender or jasmine essential oil or a control stimulus was inhaled intermittently (first $20 \mathrm{~s}$ of each 1-min interval) to prevent olfactory fatigue. The control stimulus was provided by triethyl citrate, an odourless solvent. In addition to subjective psychological assessments using a visual analogue scale, the temperature at the tip of the nose and cardiac activity on electrocardiogram were recorded as indices of peripheral and cardiac autonomic nervous system activities, respectively. Significant decreases in nose tip temperature and high-frequency (HF) component of heart rate variability (HRV), and a significant increase in heart rate were observed under all three conditions. However, compared with the control condition, lavender inhalation induced significantly greater decreases in nose tip temperature $(p<0.01)$ and HF component of HRV $(p<0.01)$, which indicated greater enhancement of sympathetic nervous system activity and suppression of parasympathetic nervous system activity. On the other hand, lavender inhalation induced a positive mood, less subjective stress, and increased concentration during the task $(p<0.01)$. These contradictory results of enhanced physiological stress response and lower subjective stress induced by lavender inhalation under stressful conditions suggest that lavender aroma may have effects other than sedation. Further studies are necessary to further clarify these effects.
\end{abstract}

Keywords: lavender, jasmine, stress response, nose temperature, olfactometer.

Adv Biomed Eng. 5: pp. 7-12, 2016.

\section{Introduction}

Aromatherapy is one of the major facets of complementary and alternative medicine worldwide. Lavender, the most commonly used aroma in aromatherapy, functions as an effective medicament for neurological disorders [1], improves task performance [2], decreases blood pressure [3] and improves sleep quality [4]. On the other hand, jasmine, which is considered a stimulating aroma, induces autonomic arousal, such as enhancement of systolic and diastolic blood pressure [5].

However, the reported efficacy of these aromas is inconsistent among studies, with frequently discrepant conclusions. Two recent studies reported that lavender relieved job-related stress among nurses [6] and ameliorated depression, anxiety and stress among pregnant women [7], whereas another study published in the same year reported no significant psychological or physiological effects of lavender aroma inhalation after coronary artery bypass surgery [8]. These studies reported conflicting results despite being well-designed, placebo-controlled studies with an adequate

This study was presented at the Symposium on Biomedical Engineering 2015, Okayama, September, 2015.

Received on July 26, 2015; revised on October 23, 2015; accepted on December 17, 2015.

* Department of Information and Management Systems Engineering, Nagaoka University of Technology, Nagaoka, Japan.

** Corporate R\&D division, Takasago International Corporation, Hiratsuka, Japan.

\# 1603-1 Kamitomioka, Nagaoka, Niigata 940-2188, Japan.

E-mail: nomura@kjs.nagaokaut.ac.jp sample size $(\mathrm{n}=60-141)$.

The discrepancy in conclusions among studies is frequently attributed to differences in the dose and duration of aroma inhalation. However, because most previous studies used an aroma container or diffuser that emits fragrant vapour at ambient temperatures or a mask-like facial cover impregnated with the aroma to deliver the aroma to subjects, control of the dose and duration of inhalation was practically difficult. Eventually, possible interference by olfactory fatigue limits the outcomes in such situations.

In this study, we used a proprietary olfactometer that precisely controls the dose and duration of inhalation to evaluate the effects of lavender and jasmine aroma under stressful conditions.

The temperature at the tip of the nose during a task was selected as the primary outcome. The peripheral skin temperature changes with a change in subcutaneous blood flow, and peripheral blood flow is solely controlled by the sympathetic nervous system. Therefore, the skin temperature at the site of interest can be measured as an index of sympathetic nervous system activity $[9$, 10]. In particular, the temperature at the tip of the nose has been used as an objective measure of psychological stress [11-15]. To our knowledge, few olfactory studies have assessed skin temperature to estimate peripheral autonomic nervous system activity.

Other outcomes included the heart rate variability (HRV) as an index of cardiac autonomic nervous system activity and subjective outcome assessed using the visual analogue scale (VAS). These parameters were commonly used in previous aroma studies.

In summary, the aim of this study was to investigate the effects of two prominent aromas, lavender and jasmine, on peripheral and cardiac autonomic nervous system activities under stress- 
ful conditions, in a highly reproducible manner using an olfactometer.

\section{Methods}

\subsection{Subjects}

Seventeen male university students with a mean age of $22.1 \pm$ 1.03 years and mean body mass index of $22.3 \pm 5.05 \mathrm{~kg} / \mathrm{m}^{2}$ were recruited for this study. All subjects were healthy with a normal olfactory function.

This study was conducted in accordance with the ethical principles of the Helsinki Declaration and after obtaining informed consent from each subject. The study was approved by the ethics committee of Nagaoka University of Technology.

\subsection{Experimental procedure}

The experiment was conducted using a within-subjects design (Fig. 1). Following a 10-min initial rest period as the initialization period (hereafter denoted as R1), each subject was instructed to perform a 30-min calculation task to induce cognitive stress (denoted as T), followed by a 15 -min recovery period (denoted as $\mathrm{R} 2)$.

The calculation task comprised simple mental calculations similar to those used in the so-called Kraepelin test, in which subjects were required to continuously add single-digit numbers displayed on a computer. All subjects were instructed to perform the task as fast and as accurately as possible. Although the calculations are very simple, the task requires sustained concentration and attention. Therefore, this task is frequently used to induce acute stress in various studies $[12,14,16]$.

Lavender essential oil (French, Takasago International Corporation, Japan) and jasmine essential oil (Moroccan, Takasago International Corporation, Japan) were prepared at $10 \mathrm{wt} \%$ with the odourless solvent triethyl citrate (TEC). TEC was also used as the control stimulus in this study. Lavender or jasmine aroma or TEC was intermittently delivered (20 s of each 1-min interval) during the task via a cannula connected to a customised olfactometer (Matsumikagakukeisoku Co. Ltd., Japan) placed under the nostrils. Intermittent delivery prevented olfactory fatigue. During the $40 \mathrm{~s}$ followed by $20 \mathrm{~s}$ of odor inhalation, there was no air or TEC flow from the olfactometer. The subjects were not given any specific instructions regarding their breathing cycles throughout the experimental period.

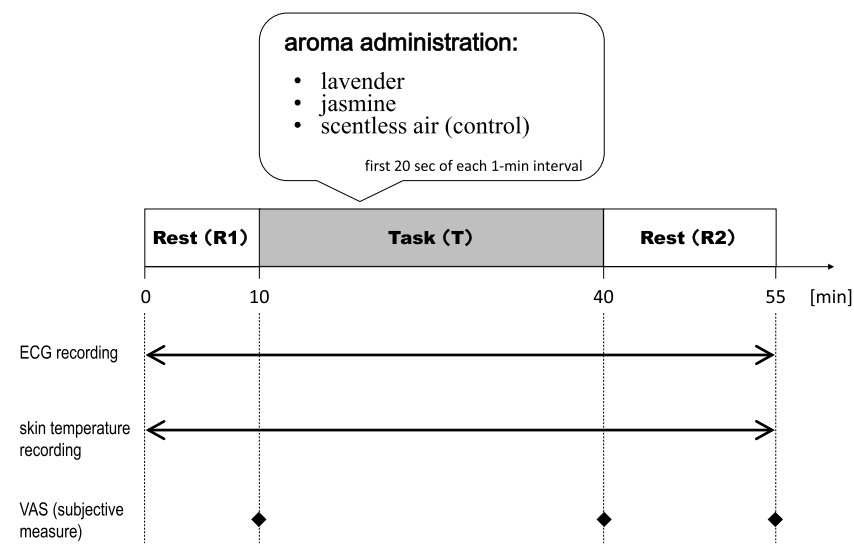

Fig. 1 Schematic presentation of the protocol.
All experiments were conducted in an air-controlled laboratory with a mean temperature of $25.1 \pm 0.8^{\circ} \mathrm{C}$ and humidity of $58.9 \pm 6.4 \%$. Each subject performed the task with each of the test and control stimulus on separate days. The sequence of aroma presentation was counter-balanced.

\subsection{Measurements}

\subsubsection{Subjective assessment of psychological parameters}

The subjects were requested to complete a VAS with seven items: frustration, tension, concentration, monotonous, effort, fatigue, and boredom, at the end of R1, at the end of $\mathrm{T}$, and at the end of R2 (Fig. 1).

The VAS was a calibrated line with two end points ( 0 and $100 \%)$. The subjects marked on the line the point that represented their perception regarding each of the seven items. Each marking required less than $10 \mathrm{~s}$.

\subsubsection{Objective assessment of physiological measures}

During each experiment (R1-T-R2), the temperature at the tip of the nose and that at the forehead (reference) were measured using a thermistor probe (ITP082-25, NIKKISO-THERM Co., Ltd., Japan), and recorded using a data logger at a sampling rate of $1.0 \mathrm{~Hz}$.

In addition, an electrocardiogram (ECG) was recorded throughout the experiment (R1-T-R2) using a bio-amplifier (MP150, BIOPAC Systems Inc., USA) at a sampling rate of $200 \mathrm{~Hz}$ of and resolution of 16 bit. The ECG data were used to analyse heart rate and HRV, which is a frequency domain of the heartbeat in a time series. The high-frequency component (HF component; $0.15-0.40 \mathrm{~Hz}$ ) of HRV represents cardiac parasympathetic nervous system activity [17].

\subsection{Statistics}

Paired t-tests and Wilcoxon signed-rank tests were performed for comparisons of objective and subjective parameters, respectively, within conditions. In case of multiple comparisons among conditions, Bonferroni correction was applied to both tests. The level of statistical significance $(p)$ was set at 0.05 .

\section{Results}

\subsection{Task performance}

There was no difference in the performance of the task, irrespective of the condition, probably because the addition of single-digit numbers was too simple to result in performance differences.

\subsection{VAS scores}

The changes in VAS scores from $\mathrm{R} 1$ to $\mathrm{T}$ and from $\mathrm{T}$ to $\mathrm{R} 2$ are shown in Table 1.

Under the control condition, the VAS scores for frustration, effort, fatigue and boredom were significantly higher at $\mathrm{T}$ than at R1 ( $p<0.001$ to $<0.05)$, indicating that the simple calculation task functioned as a stressor, as reported previously $[12,14,16]$.

With lavender inhalation, the VAS scores for fatigue and boredom remained unchanged at $\mathrm{R} 1$ and $\mathrm{T}$, while those for frustration, concentration and effort were higher at T than at R1 $(p<$ 0.001 to < 0.01). With jasmine inhalation, the VAS scores for frustration, effort, fatigue, boredom, concentration and tension were significantly higher at $\mathrm{T}$ than at $\mathrm{R} 1(p<0.001$ to $<0.05)$.

Among the three conditions, the VAS score for concentration 
Table 1 Results [mean (SD)] of subjective measures (VAS).

\begin{tabular}{|c|c|c|c|c|}
\hline & \multicolumn{2}{|c|}{$\Delta(\mathbf{R} 1-\mathrm{T})$} & \multicolumn{2}{|c|}{$\Delta(\mathrm{T}-\mathrm{R} 2)$} \\
\hline \multicolumn{5}{|l|}{ frustration } \\
\hline control & 25.0 & $(23.5)^{* * *}$ & -15.4 & $(22.6)^{*}$ \\
\hline lavender & 17.2 & $(21.8)^{* *}$ & -7.2 & (24.5) \\
\hline jasmine & 17.0 & $(25.6)^{*}$ & -5.1 & (22.2) \\
\hline \multicolumn{5}{|l|}{ tension } \\
\hline control & 12.4 & $(32.5)$ & -17.2 & $(28.7)^{*}$ \\
\hline lavender & 11.2 & $(24.7)$ & -22.4 & $(25.2)^{* *}$ \\
\hline jasmine & 21.5 & $(31.3)^{*}$ & -24.5 & $(28.3)^{* *}$ \\
\hline \multicolumn{5}{|c|}{ concentration } \\
\hline control & 5.8 & $(30.9)^{\# \#}$ & -19.7 & $(21.5)^{* *}$ \\
\hline lavender & 16.3 & $(23.0)^{* *, \# \#}$ & -34.0 & $(28.8)^{* * *}$ \\
\hline jasmine & 22.4 & $(38.6)^{*}$ & -23.7 & $(28.1)^{* *}$ \\
\hline \multicolumn{5}{|c|}{ monotonous } \\
\hline control & 4.4 & $(22.3)$ & 8.0 & (20.3) \\
\hline lavender & -9.2 & (19.8) & 18.4 & $(27.3)^{*}$ \\
\hline jasmine & -5.3 & (20.1) & 17.1 & $(27.3)^{*}$ \\
\hline \multicolumn{5}{|l|}{ effort } \\
\hline control & 19.9 & $(28.9)^{*}$ & -23.0 & $(29.5)^{* *}$ \\
\hline lavender & 28.4 & $(26.7)^{* * * *}$ & -28.6 & $(33.3)^{* *}$ \\
\hline jasmine & 29.5 & $(27.1)^{* * * *}$ & -26.8 & $(33.7)^{* *}$ \\
\hline \multicolumn{5}{|l|}{ fatigue } \\
\hline control & 20.9 & $(33.1)^{*}$ & -12.9 & $(24.9)^{*}$ \\
\hline lavender & 18.4 & (37.4) & -7.1 & (22.8) \\
\hline jasmine & 22.9 & $(27.4)^{* * *}$ & -12.5 & (30.7) \\
\hline \multicolumn{5}{|l|}{ boredom } \\
\hline control & 19.8 & $(24.4)^{* *}$ & -9.3 & (26.9) \\
\hline lavender & 11.0 & $(26.1)$ & -4.3 & (28.9) \\
\hline jasmine & 13.1 & $(19.5)^{*}$ & -7.2 & (14.3) \\
\hline
\end{tabular}

at $\mathrm{T}$ was significantly higher with lavender inhalation than with the control stimulus $(p<0.01)$. No other significant differences were observed among the three conditions.

\subsection{Nose tip temperature}

Figure 2 shows the changes in temperature at the tip of the nose relative to the temperature at the forehead as reference (defined as the difference between the nose and forehead temperatures). Note that the raw values are standardised [so-called z-score; mean $(\mu)$ and standard deviation $(\sigma)$ for the population $(\mathrm{x})$ transformed to 0.0 and 1.0 by the formula $\mathrm{z}=(\mathrm{x}-\mu) / \sigma]$ with respect to each subject and condition because of the large individual variations. The baseline value for each condition, denoted by the mean value obtained at R1, was equalised among the conditions as 0.0 .

Regardless of the aroma condition, the nose tip temperature was significantly lower at T than at $\mathrm{R} 1(p<0.001$ to $<0.01)$, and was restored to normal at $\mathrm{R} 2(p<0.001$ to $<0.05)$. These changes indicate a typical acute stress response $[12,14]$.

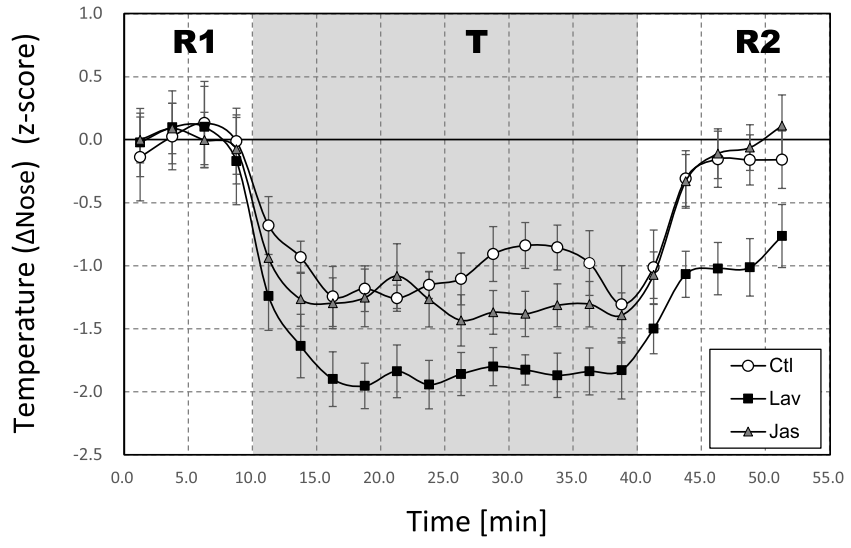

Fig. 2 Changes in nose temperature (mean \pm SEM per 2.5 min).

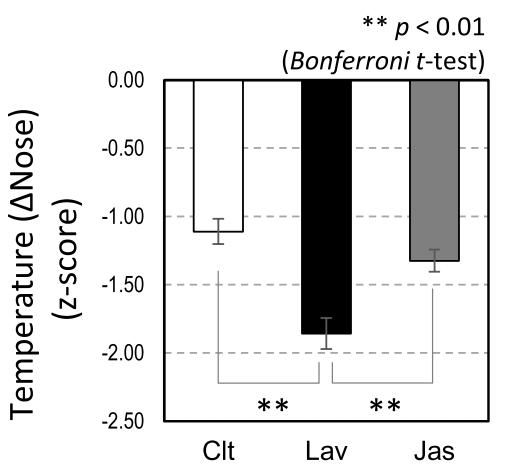

Fig. 3 Average changes in nose temperature during the task (mean \pm SEM).

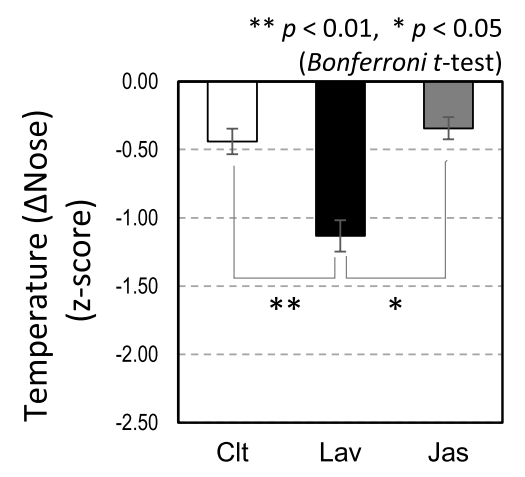

Fig. 4 Average changes in nose temperature during rest after the task $($ mean \pm SEM $)$.

The mean nose tip temperature at $\mathrm{T}$ was significantly lower with lavender inhalation than with control $(p<0.01)$ or jasmine $(p<0.01)$ inhalation (Fig. 3). Similar results were obtained for the temperature at R2 $(p<0.01$ vs. control condition and $p<0.05$ vs. jasmine condition; Fig. 4). These results indicate that the decrease in blood flow at the tip of the nose during the task was significantly greater under the lavender inhaling condition than under the control and jasmine inhaling conditions. 
(a)

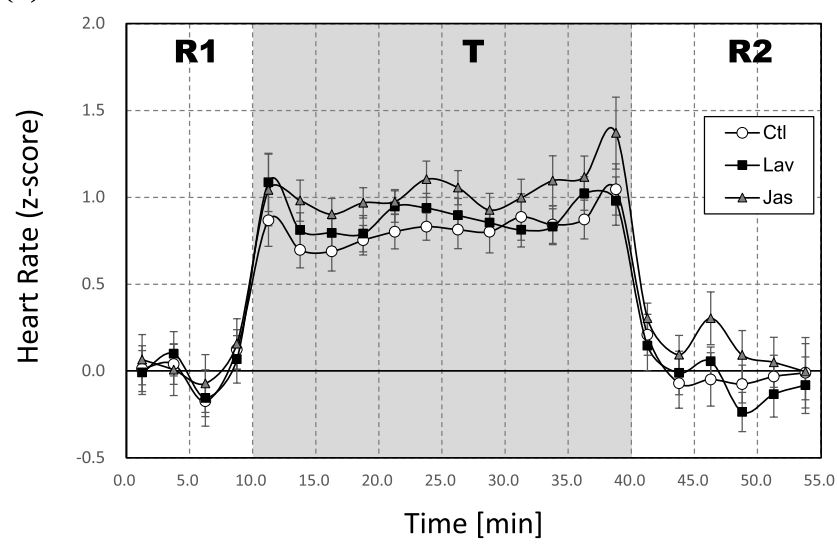

(b)

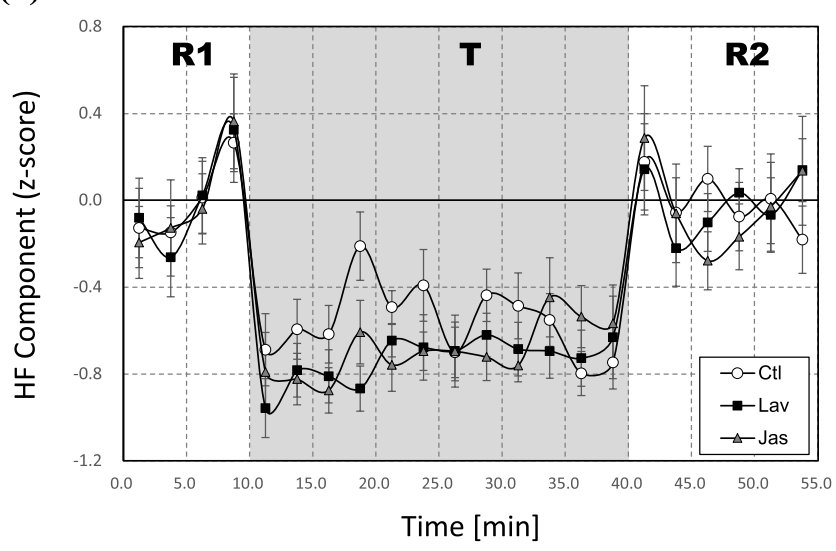

Fig. 5 Changes in (a) heart rate and (b) high-frequency component of heart rate variability (mean \pm SEM per $2.5 \mathrm{~min}$ ).

\subsection{Heart rate and HRV}

Figure $\mathbf{5 a}$ and $\mathbf{5 b}$ show the heart rate and the HF component of HRV for the three conditions. The same standardisation and baseline correction procedure used for the nose tip temperature were applied to these data.

Regardless of the aroma condition, heart rate was significantly higher at $\mathrm{T}$ than at $\mathrm{R} 1(p<0.0001)$, and was restored to normal at R2 $(p<0.0001)$. The HF component of HRV was significantly lower at $\mathrm{T}$ than at $\mathrm{R} 1(p<0.001$ to $<0.01)$, while it was restored to normal at $\mathrm{R} 2(p<0.001$ to $<0.01)$. These findings represent a typical acute stress response [14].

The mean heart rate at $\mathrm{T}$ was significantly higher under the jasmine inhaling condition than under the control condition $(p<$ 0.01 ; Fig. 6), indicating increased cardiac activity by jasmine inhalation during the task. Although a trend of higher mean heart rate at $\mathrm{T}$ was observed for the lavender condition compared with the control condition, the difference was not statistically significant $(p<0.10$; Fig. 6).

The HF component of HRV at T was significantly lower under the lavender inhaling condition than under the control condition $(p<0.05)$, with the decrease induced by lavender inhalation being more prominent in the first half of the task period $(p<0.01$; Fig. 7). This represents increased suppression of parasympathetic nervous system activity by lavender inhalation during the task.

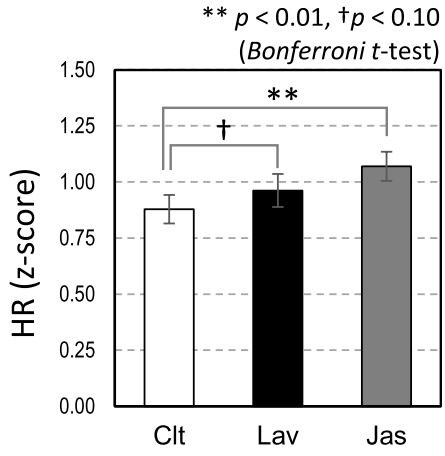

Fig. 6 Average changes in heart rate during the task (mean \pm SEM).

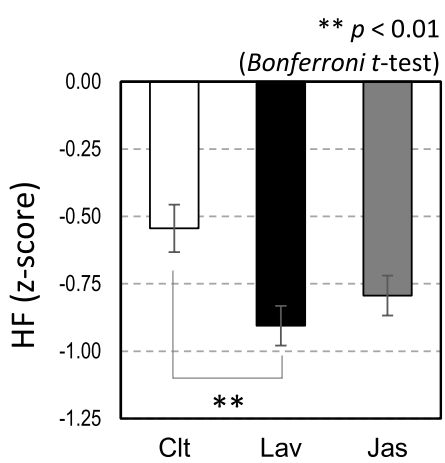

Fig. 7 Average changes in the HF component during the first half of the task period (mean $\pm \mathrm{SEM}$ ).

\section{Discussion}

In this study, we investigated the effects of two prominent aromas, lavender and jasmine, on peripheral and cardiac autonomic nervous system activities under stressful conditions, in a highly reproducible manner using an olfactometer. The peripheral and cardiac responses during the task period and the subjective findings under the control condition collectively indicated that the calculation task employed in this study functioned as an acute stressor. The decrease in peripheral skin temperature during the task, which represents a decrease in blood flow, indicates enhanced peripheral sympathetic nervous system activity; the increase in HR indicates enhanced cardiac sympathetic nervous system activity; and the decrease in the HF component of HRV indicates decreased cardiac parasympathetic nervous system activity. Such hemodynamic change or re-allocation of blood in the body is frequently observed as part of the physiological stress response to acute cognitive stressors and is considered to be an adaptive response, i.e. re-allocation of blood to foster brain functioning for problem solving [18, 19].

Significant decreases in nose tip temperature and HF component of HRV and a significant increase in heart rate were observed in all three conditions in this study, although the changes in nose tip temperature and HF component of HRV were more marked in the lavender condition than in the control condition. The lavender aroma has been reported to function as a sedative that activates the parasympathetic nervous system. However, the findings of this study indicate that lavender aroma inhalation increases stress. 
On the other hand, the results of subjective evaluations, which showed no changes in the VAS score for fatigue and boredom and a higher score for concentration with lavender inhalation, suggest that the enhanced physiological stress response with lavender inhalation may not be a result of greater psychological stress.

To our knowledge, few studies have shown such discrepant effects of lavender inhalation on the mind and body. Nevertheless, Field et al. [2] reported improved performance in a calculation task under lavender aroma inhalation, while Heuberger et al. [20] reported improved performance in a cognitive task (vigilance task) under inhalation of lialyl-acetate, a major compound of lavender essential oil. Our findings seem to be consistent with the findings of these behavioral studies. Collectively, we can conclude that the lavender aroma may work as a fortifier, not a sedative, under stressful conditions by inducing an adaptive response of enhancing blood re-allocation.

The biological mechanism underlying the discrepant effects of lavender inhalation on the mind and body remains unclear. Takahashi et al. [21] used mouse models and reported that lavender inhalation had a bi-directional influence on the central nervous system of the animals, either attenuating the effects of stress or functioning as a stressor under high or low stress loads. A similar bi-directional efficacy may exist for humans, depending on the condition. Further biological studies are necessary for a better understanding of these complex effects.

With regard to jasmine inhalation, the increase in HR was greater than that with control inhalation, indicating greater enhancement of sympathetic nervous system activity. However, in contrast to lavender, jasmine did not work positively on the mind. We speculate that the increase in sympathetic nervous system activity with jasmine inhalation may be an extension of the ordinary stress response.

This study is limited by the inclusion of a homogenous study population (male university students), a similar concentration of the jasmine and lavender essential oils (10 wt \%) and a task with no variations in the difficulty level. Further studies focusing on these parameters and using a variety of aromas in a similar experimental setting will aid in the further understanding of the benefits of aromatherapy.

\section{Conclusions}

In conclusion, lavender inhalation induced a relatively greater physiological stress response but lower VAS scores for subjective stress-related symptoms under stressful conditions. These results suggest that the lavender aroma may have functions other than sedation.

\section{Acknowledgement}

The authors declare no conflict of interest associated with this manuscript.

\section{References}

1. Koulivand PH, Khaleghi Ghadiri M, Gorji A: Lavender and the nervous system. Evid Based Complement Alternat Med. 2013, pp. 1-10, 2013.

2. Field T, Diego M, Hernandez-Reif M, Cisneros W, Feijo L, Vera Y, Gil K, Grina D, Claire He Q: Lavender fragrance cleansing gel effects on relaxation. Int J Neurosci. 115(2), pp. 207-222, 2005.

3. Kim IH, Kim C, Seong K, Hur MH, Lim HM, Lee MS: Essential oil inhalation on blood pressure and salivary cortisol levels in prehypertensive and hypertensive subjects. Evid Based Complement Alternat Med. 2012, pp. 1-9, 2013.

4. Lillehei AS, Halcon LL: A systematic review of the effect of inhaled essential oils on sleep. J Altern Complement Med. 20(6), pp. 441-451, 2014.

5. Hongratanaworakit T: Stimulating effect of aromatherapy massage with jasmine oil. Nat Prod Commun. 5(1), pp. 157-162, 2010.

6. Chen MC, Fang SH, Fang L: The effects of aromatherapy in relieving symptoms related to job stress among nurses. Int J Nurs Pract. 21, pp. 87-93, 2015.

7. Effati-Daryani F, Mohammad-Alizadeh -Charandabi S, Mirghafourvand M, Taghizadeh M, Mohammadi A: Effect of Lavender cream with or without foot-bath on anxiety, stress and depression in pregnancy: a randomized placebo-controlled trial. J Caring Sci. 4(1), pp. 63-73, 2015.

8. Bikmoradi A, Seifi Z, Poorolajal J, Araghchian M, Safiaryan R, Oshvandi K: Effect of inhalation aromatherapy with lavender essential oil on stress and vital signs in patients undergoing coronary artery bypass surgery: A single-blinded randomized clinical trial. Complement Ther Med. 23(3), pp. 331-338, 2015.

9. Nozawa A, Takei Y: Dynamic analysis of dorsal thermal image. Artif Life Robotics. 16(2), pp. 147-151, 2011.

10. Nozawa A, Tomono S, Mizuno T, Ide H: Detection of fight or flight reaction on facial skin thermogram using spatio-temporal spectrum differential analysis. IEEJ Trans FM. 126(6), pp. 470477, 2006.

11. Zenju H, Nozawa A, Tanaka H, Ide H: Estimation of unpleasant and pleasant states by nasal thermogram. IEEJ Trans EIS. 124(1), pp. 213-214, 2004.

12. Hioki K, Nozawa A, Mizuno T, Ide H: Physiological evaluation of mental workload in time pressure. IEEJ Trans EIS. 127(7), pp. 1000-1006, 2007.

13. Nozawa A, Tacano M: Correlation analysis on alpha attenuation and nasal skin temperature. J Stat Mech. 2009, pp. 1-10, 2009.

14. Mizuno T, Nomura S, Nozawa A, Asano H, Ide H: Evaluation of the effect of intermittent mental work-load by nasal skin temperature. IEICE Trans IS. 93(4), pp. 535-543, 2010.

15. Mizuno T, Nakategawa N, Kume Y: Color influences on human beings evaluated by nasal skin temperature. Artif Life Robotics. 16(4), pp. 519-522, 2012.

16. Nomura S, Mizuno T, Nozawa A, Ide H: Characteristics of salivary chromogranin $\mathrm{A}$ as a short-term mental stress biomarker. Trans Japan Soc Med Biol Eng. 48(2), pp. 207-212, 2010.

17. Pomeranz B, Macaulay RJ, Caudill MA, Kutz I, Adam D, Gordon D, Kilborn KM, Barger AC, Shannon DC, Cohen RJ, Benson $\mathrm{H}$ : Assessment of autonomic functions in human by heart rate spectral analysis. Am J Physiol. 248(1), pp. 151-153, 1985.

18. Obrist PA: Cardiovascular psychophysiology: A perspective. Plenum Press, New York, 1981.

19. Williams RB: Patterns of reactivity and stress: In Handbook of stress, reactivity, and cardiovascular disease. John Wiley \& Sons, New York, 1986.

20. Heuberger E, Ilmberger J: The influence of essential oils on human vigilance. Nat Prod Commun. 5(9), pp. 1441-1446, 2010.

21. Takahashi M, Yoshino A, Yamanaka A, Asanuma C, Satou T, Hayashi S, Masuo Y, Sadamoto K, Koike K: Effects of inhaled lavender essential oil on stress-loaded animals: change in anxiety-related behavior and expression levels of selected mRNAs 
and proteins. Nat Prod Commun. 7(11), pp. 1539-1544, 2012.

\section{Shusaku Nomura}

Shusaku NomURa is an Associate Professor at Nagaoka University of Technology, Japan. He received his Ph.D in Science from the Graduate School of Science and Technology, Kobe University, in 2001. His research interests include Ambient Biomedical Engineering, Psychoneuroendocrinology, and Kansei Physiology.

\section{Kento Maevama}

Kento MaEyama is a master course student in the faculty of Management and Information Systems Engineering, Nagaoka University of Technology, Japan. His research interests include Ambient Biomedical Engineering.
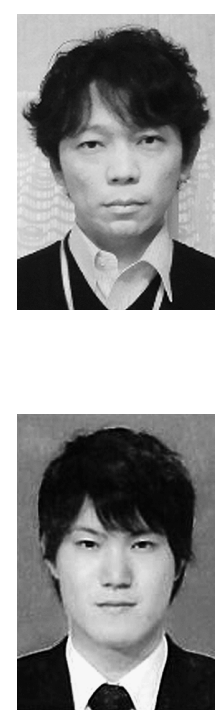

\section{Kanetoshi Iто}

Kanetoshi Iто received the B.S. degree in agriculture degree from University of the Ryukyus, Japan, in 2006 and the M.D. degree in science from Tokyo Metropolitan University, Japan, in 2008. He is working for Takasago International Corporation, Japan, from 2008. His current interests include the

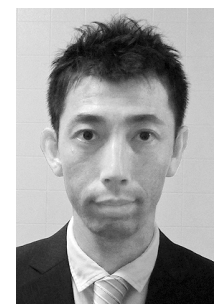
endocrinology, bio-signal processing, functional neuroimaging and cognitive neuroscience. He is member of Association for Chemoreception Sciences and Human Brain Mapping Society. 